PROCEEDINGS OF THE

AMERICAN MATHEMATICAL SOCIETY

Volume 130, Number 3, Pages 667-672

S 0002-9939(01)06193-7

Article electronically published on August 29, 2001

\title{
GROUP GENERATED BY THE WEIERSTRASS POINTS OF A PLANE QUARTIC
}

\author{
MARTINE GIRARD AND PAVLOS TZERMIAS \\ (Communicated by David E. Rohrlich)
}

\begin{abstract}
We describe the group generated by the Weierstrass points in the Jacobian of the curve $X^{4}+Y^{4}+Z^{4}+3\left(X^{2} Y^{2}+X^{2} Z^{2}+Y^{2} Z^{2}\right)=0$. This curve is the only curve of genus 3, apart from the fourth Fermat curve, possessing exactly twelve Weierstrass points.
\end{abstract}

Let $\mathcal{C}$ be a smooth projective curve of genus $g$. For $D$ a divisor, let $[D]$ be its class in $\operatorname{Pic}^{0}(\mathcal{C})$. Let $J$ be the Jacobian of $\mathcal{C}$ which we identify with $\operatorname{Pic}^{0}(\mathcal{C})$. We choose a Weierstrass point $\infty$ as base point for the Abel-Jacobi map

$$
\begin{array}{llc}
\mathcal{C} & \rightarrow & J \\
P & \mapsto[P-\infty]
\end{array}
$$

which can be extended linearly to divisors $\operatorname{Div}(\mathcal{C})$.

We study the structure of the group $W$ generated by the images under $j$ of Weierstrass points in the Jacobian of $\mathcal{C}$. This structure does not depend on the Weierstrass point chosen as base-point for the map $j$ and thus provides an interesting geometric invariant.

The simplest case is the case of a hyperelliptic curve, where it is easy to see that $W=(\mathbb{Z} / 2 \mathbb{Z})^{2 g}=J[2]$ (see, for instance, [5]).

For non-hyperelliptic curves of genus 3 - that is, plane quartics - some particular cases have already been considered. Each of these curves has a large automorphism group. More precisely, for the Klein curve (given by $X^{3} Y+Y^{3} Z+Z^{3} X=0$ ), with 168 automorphisms, $W=(\mathbb{Z} / 2 \mathbb{Z}) \times(\mathbb{Z} / 7 \mathbb{Z})^{3}[\underline{6}$. For the Fermat quartic (given by $\left.X^{4}+Y^{4}=Z^{4}\right)$, with 96 automorphisms, $W=(\mathbb{Z} / 4 \mathbb{Z})^{5} \times(\mathbb{Z} / 2 \mathbb{Z})[7$. For the Picard curve (given by $\left.Y^{3} Z+Z^{4}=X^{4}\right)$, with 48 automorphisms, $W=(\mathbb{Z} / 4 \mathbb{Z})^{2} \times(\mathbb{Z} / 3 \mathbb{Z})^{5}$ [2]. Finally, in [1], a family of smooth curves of genus 3 is determined for which $W=\mathbb{Z}^{4} \times(\mathbb{Z} / 3 \mathbb{Z})^{5}$.

In this note, we consider the smooth curve $\mathcal{C}$ of genus 3 given by the following equation:

$$
X^{4}+Y^{4}+Z^{4}+3\left(X^{2} Y^{2}+X^{2} Z^{2}+Y^{2} Z^{2}\right)=0 .
$$

This curve has 24 automorphisms [4]. We prove the following result:

Theorem 0.1. Let $W$ be the group generated by the images of the Weierstrass points in the Jacobian $J$ of $\mathcal{C}$. Then $W \cong(\mathbb{Z} / 4 \mathbb{Z})^{5}$.

Received by the editors September 18, 2000.

1991 Mathematics Subject Classification. Primary 11G30, 14H25; Secondary 14H45.

Key words and phrases. Algebraic curves, Jacobians, Weierstrass points.

(C)2001 American Mathematical Society 
This curve possesses exactly 12 Weierstrass points, each one of weight 2 . There are only two smooth curves of genus 3 with exactly 12 Weierstrass points, the Fermat quartic and the one we are considering [4].

In order to prove Theorem 0.1 we use geometric arguments to reduce the number of generators of $W$. Then, since the Jacobian of this curve is isogenous to the product of three copies of the same elliptic curve $\mathcal{E}$, the study of the images of those points in $\mathcal{E}$ under the three morphisms from $\mathcal{C}$ to $\mathcal{E}$ enables us to determine exactly the structure of $W$.

\section{Computation of the Weierstrass points}

In the case of a smooth quartic, the Weierstrass points are its flexes. They are the zeroes of the Hessian $H$ given by

$$
\begin{aligned}
H(X, Y, Z)= & 2 X^{6}+\left(3 Z^{2}+3 Y^{2}\right) X^{4}+\left(8 Y^{2} Z^{2}+3 Z^{4}+3 Y^{4}\right) X^{2} \\
& +2 Z^{6}+2 Y^{6}+3 Z^{4} Y^{2}+3 Z^{2} Y^{4}
\end{aligned}
$$

hence the zeroes of $\left(X^{2}+Y^{2}\right)\left(Y^{2}+Z^{2}\right)\left(Z^{2}+X^{2}\right)$. The Weierstrass points are thus the intersection points of the curve with the 6 lines given by the following equations: $L_{1}: X=i Y, L_{2}: X=-i Y, L_{3}: Y=i Z, L_{4}: Y=-i Z, L_{5}: Z=i X$, $L_{6}: Z=-i X$. More precisely, the Weierstrass points are distributed on the $L_{i}$ 's as follows:

- On $L_{1}: P_{1,1}=(i, 1,1), P_{1,2}=(-i,-1,1), P_{1,3}=(-1, i, 1), P_{1,4}=(1,-i, 1)$.

- On $L_{2}: P_{2,1}=(-i, 1,1), P_{2,2}=(i,-1,1), P_{2,3}=(1, i, 1), P_{2,4}=(-1,-i, 1)$.

- On $L_{3}: P_{1,3}, P_{2,3}, P_{3,3}=(-i, i, 1), P_{3,4}=(i, i, 1)$.

- On $L_{4}: P_{1,4}, P_{2,4}, P_{4,3}=(-i,-i, 1), P_{4,4}=(i,-i, 1)$.

- On $L_{5}: P_{1,2}, P_{2,1}, P_{3,3}, P_{4,3}$.

- On $L_{6}: P_{1,1}, P_{2,2}, P_{3,4}, P_{4,4}$.

Moreover, the curve meets the tangent line at any of those points with intersection multiplicity equal to 4 . This immediately yields relations between the images of Weierstrass points in the Jacobian $J$. Let's choose the point $P_{3,3}$ as base point for the Abel-Jacobi map. The embedding $j$ in the Jacobian is $P \mapsto\left[P-P_{3,3}\right]$. By abuse of notation, we denote in the same way a point and its image under $j$. That means that we will denote by $D=0$ the fact that a divisor class $[D]$ is in the kernel of $j$.

Under this convention, we have

- $4 P_{i, j}=0$.

- $P_{1,1}+P_{1,2}+P_{1,3}+P_{1,4}=0$.

- $P_{2,1}+P_{2,2}+P_{2,3}+P_{2,4}=0$.

- $P_{1,3}+P_{2,3}+P_{3,3}+P_{3,4}=0$.

- $P_{1,4}+P_{2,4}+P_{4,3}+P_{4,4}=0$.

- $P_{1,2}+P_{2,1}+P_{3,3}+P_{4,3}=0$.

- $P_{1,1}+P_{2,2}+P_{3,4}+P_{4,4}=0$.

These results enable us to write $P_{1,4}, P_{2,4}, P_{3,4}, P_{4,3}$ and $P_{4,4}$ in terms of $P_{1,1}$, $P_{1,2}, P_{1,3}, P_{2,1}, P_{2,2}, P_{2,3}$ and $P_{3,3}$ (which is zero in $J$ ). Moreover, by Lemma 4.1 in Section 4 , we have the additional relation $P_{1,2}+P_{2,2}-P_{1,1}-P_{2,1}=2 P_{1,3}-2 P_{2,3}$. Therefore, we can also express $P_{2,2}$ in terms of $P_{1,1}, P_{1,2}, P_{1,3}, P_{2,1}$ and $P_{2,3}$. We can thus conclude that

Proposition 1.1. $W$ is a quotient of $(\mathbb{Z} / 4 \mathbb{Z})^{5}$. 
We will show that $W$ is isomorphic to this group; that means that there are no relations between the Weierstrass points other than the ones we already found. The proof will rely on the fact that the Jacobian is isogenous to the product of three copies of the same elliptic curve [3].

\section{Structure of the Jacobian of $\mathcal{C}$}

Let $\mathcal{D}$ be the affine curve given by the equation $x^{4}+y^{2}+1+3\left(x^{2} y+x^{2}+y\right)=0$. There is a birational map from this curve to the elliptic curve $\mathcal{E}$ given by the affine equation $v^{2}=u(u-1 / 4)(u-5 / 4)$. This morphism, denoted by $\psi$, is given by

$$
(x, y) \mapsto\left(u=\frac{1}{4} \frac{4 y+11 x^{2}+2 x+11}{(x+1)^{2}}, v=-\frac{1}{4} \frac{(x-1)\left(4 y+11 x^{2}+2 x+11\right)}{(x+1)^{3}}\right) .
$$

Let $\phi_{1}, \phi_{2}$ and $\phi_{3}$ be the three morphisms from $\mathcal{C}$ to $\mathcal{D}$ given by

$$
\left\{\begin{aligned}
& \phi_{1}:(X, Y, Z) \mapsto\left(\frac{X}{Z},\left(\frac{Y}{Z}\right)^{2}\right), \\
& \phi_{2}:(X, Y, Z) \mapsto\left(\frac{Y}{X},\left(\frac{Z}{X}\right)^{2}\right), \\
& \phi_{3}:(X, Y, Z) \mapsto\left(\frac{Z}{Y},\left(\frac{X}{Y}\right)^{2}\right) .
\end{aligned}\right.
$$

Proposition 2.1. The Jacobian of $\mathcal{C}$ is isogenous to $\mathcal{E} \times \mathcal{E} \times \mathcal{E}$.

Proof. When $\omega$ is a nonzero differential form on $\mathcal{D}$, it's easy to check that $\omega_{1}=$ $\phi_{1}^{\star}(\omega), \omega_{2}=\phi_{2}^{\star}(\omega)$ and $\omega_{3}=\phi_{3}^{\star}(\omega)$ are independent differential forms on $\mathcal{C}$. More precisely, if

$$
\omega=\frac{d x}{y+\frac{3}{2} x^{2}+\frac{3}{2}}
$$

then

$$
\begin{gathered}
\omega_{1}=\frac{d x}{y^{2}+\frac{3}{2} x^{2}+\frac{3}{2}}, \\
\omega_{2}=\frac{d\left(\frac{y}{x}\right)}{\left(\frac{1}{x}\right)^{2}+\frac{3}{2} \frac{y^{2}}{x^{2}}+\frac{3}{2}}=\frac{x d y-y d x}{1+\frac{3}{2} y^{2}+\frac{3}{2} x^{2}}=\frac{1}{y} \frac{d x}{y^{2}+\frac{3}{2} x^{2}+\frac{3}{2}} \\
\text { and } \omega_{3}=\frac{d\left(\frac{1}{y}\right)}{\left(\frac{x}{y}\right)^{2}+\frac{3}{2} \frac{1}{y^{2}}+\frac{3}{2}}=\frac{-d y}{x^{2}+\frac{3}{2} y^{2}+\frac{3}{2}}=\frac{x}{y} \frac{d x}{y^{2}+\frac{3}{2} x^{2}+\frac{3}{2}},
\end{gathered}
$$

since $y\left(2 y^{2}+3 x^{2}+3\right) d y+x\left(2 x^{2}+3 y^{2}+3\right) d x=0$.

\section{IMAGES ON THE ELLIPTIC CURVE}

The image of a Weierstrass point under each of the three maps $\psi \circ \phi_{j}$ is among the following points on the elliptic curve $\mathcal{E}$ :

$$
\begin{gathered}
A=\left(\frac{1}{4}-\frac{1}{2} i,-\frac{1}{2}-\frac{1}{4} i\right), \quad B=\left(\frac{1}{4}+\frac{1}{2} i,-\frac{1}{2}+\frac{1}{4} i\right), \quad C=(5 / 4,0), \\
D=\left(\frac{1}{4}-\frac{1}{2} i, \frac{1}{2}+\frac{1}{4} i\right), \quad E=\left(\frac{1}{4}+\frac{1}{2} i, \frac{1}{2}-\frac{1}{4} i\right), \quad \infty,
\end{gathered}
$$

where $\infty$ denotes the point at infinity, which we take as identity element for the group law. 
Elementary computations give us the following relations between these points on the elliptic curve $\mathcal{E}$ :

$$
\left\{\begin{array}{c}
2 C=A+D=B+E=\infty \\
2 A=2 B=2 D=2 E=\left(\frac{1}{4}, 0\right) \\
A+B=D+E=C \\
A+E=B+D=(0,0) \\
C+(0,0)=\left(\frac{1}{4}, 0\right) \\
2(0,0)=2\left(\frac{1}{4}, 0\right)=2\left(\frac{5}{4}, 0\right)=\infty
\end{array}\right.
$$

It will be useful to know from which Weierstrass points each of these particular points arises.

Since $\phi_{1}$ is a map of degree 2 and $P_{1,1}$ and $P_{2,2}$ have the same image on the curve $\mathcal{D}$, namely the point $(i, 1)$, whose image under $\psi$ is $A$, we get $\left(\psi \circ \phi_{1}\right)^{*}(A)=$ $P_{1,1}+P_{2,2}$. Similar computations yield the following table:

\begin{tabular}{|c||c|c|c|}
\hline$P$ & $\left(\psi \circ \phi_{1}\right)^{*}(P)$ & $\left(\psi \circ \phi_{2}\right)^{*}(P)$ & $\left(\psi \circ \phi_{3}\right)^{*}(P)$ \\
\hline \hline$A$ & $P_{1,1}+P_{2,2}$ & $P_{2,3}+P_{2,4}$ & $P_{4,3}+P_{4,4}$ \\
\hline$B$ & $P_{1,2}+P_{2,1}$ & $P_{1,3}+P_{1,4}$ & $P_{3,3}+P_{3,4}$ \\
\hline$C$ & $P_{1,4}+P_{2,3}$ & $P_{3,4}+P_{4,3}$ & $P_{1,1}+P_{2,1}$ \\
\hline$D$ & $P_{3,3}+P_{4,3}$ & $P_{1,1}+P_{1,2}$ & $P_{1,3}+P_{2,3}$ \\
\hline$E$ & $P_{3,4}+P_{4,4}$ & $P_{2,1}+P_{2,2}$ & $P_{1,4}+P_{2,4}$ \\
\hline$\infty$ & $P_{1,3}+P_{2,4}$ & $P_{3,3}+P_{4,4}$ & $P_{1,2}+P_{2,2}$ \\
\hline
\end{tabular}

\section{Proof of the main Result}

Lemma 4.1. The divisor $P_{1,2}+P_{2,2}-P_{1,1}-P_{2,1}-2 P_{1,3}+2 P_{2,3}$ on $\mathcal{C}$ is principal.

Proof. Since the divisor $A+D-2 \infty$ on $\mathcal{E}$ is principal, the divisor

$$
\left(\psi \circ \phi_{1}\right)^{*}(A+D-2 \infty)=P_{1,1}+P_{2,2}+P_{3,3}+P_{4,3}-2 P_{1,3}-2 P_{2,4}
$$

on $\mathcal{C}$ is also principal. The lemma follows from the relations in Section 1 , since the latter divisor is linearly equivalent to $P_{1,2}+P_{2,2}-P_{1,1}-P_{2,1}-2 P_{1,3}+2 P_{2,3}$.

Lemma 4.2. For distinct Weierstrass points $P, Q, R$ on $\mathcal{C}, 2[P+Q-2 R] \neq 0$.

Proof. It is well-known that on a smooth plane quartic the canonical linear series is cut out by lines in $\mathbb{P}^{2}$. Since $4 R$ is a canonical divisor on $\mathcal{C}$, the existence of a rational function whose divisor equals $2 P+2 Q-4 R$ would imply the existence of a bitangent line through the two flexes $P$ and $Q$. This is impossible, since the tangent line at $P$ has contact of order 4 with $\mathcal{C}$ at $P$.

Proof of Theorem 0.1. By Proposition 1.1, it suffices to show that $2 W$ is isomorphic to $(\mathbb{Z} / 2 \mathbb{Z})^{5}$. Equivalently, we need to show that the divisor classes $\left[2 P_{1,1}-2 P_{3,3}\right]$, $\left[2 P_{1,2}-2 P_{3,3}\right],\left[2 P_{1,3}-2 P_{3,3}\right],\left[2 P_{2,1}-2 P_{3,3}\right]$ and $\left[2 P_{2,3}-2 P_{3,3}\right]$ are linearly independent over $\mathbb{Z} / 2 \mathbb{Z}$. Suppose, on the contrary, that for some coefficents $c_{1}, c_{2}, c_{3}$, $c_{4}, c_{5}$ in $\mathbb{Z} / 2 \mathbb{Z}$ (not all equal to 0 ) we had

$$
\begin{aligned}
{\left[2 c_{1} P_{1,1}+2 c_{2} P_{1,2}+2 c_{3} P_{1,3}+2 c_{4} P_{2,1}+\right.} & 2 c_{5} P_{2,3} \\
& \left.-2\left(c_{1}+c_{2}+c_{3}+c_{4}+c_{5}\right) P_{3,3}\right]=0 .
\end{aligned}
$$


Let $D$ denote the left-hand side of the latter equality. Looking at $\left(\psi \circ \phi_{j}\right)(D)$, for $1 \leq j \leq 3$, and using the results in the previous section we get

$$
\left(c_{3}+c_{5}\right)\left(\frac{1}{4}, 0\right)=\left(c_{1}+c_{2}+c_{3}+c_{4}+c_{5}\right)\left(\frac{1}{4}, 0\right)=\left(c_{1}+c_{2}+c_{4}\right)\left(\frac{1}{4}, 0\right)=0
$$

therefore $c_{3}=c_{5}$ and $c_{1}+c_{2}+c_{4}=0$. If $c_{3}=c_{5}=0$ or $c_{1}=c_{2}=c_{4}=0$, then exactly two of the coefficients $c_{j}$ are equal to 1 , so, by Lemma $4.2, D \neq 0$, a contradiction. So we may assume that $c_{3}=c_{5}=1$ and exactly one of the coefficients $c_{1}, c_{2}, c_{4}$ equals 0 . We have the following three cases to consider:

Case 1. Suppose that $c_{1}=0$. Then, by the relations in Section 1, we get

$$
0=D=\left[2\left(P_{1,2}+P_{1,3}+P_{2,1}+P_{2,3}\right)-8 P_{3,3}\right]=\left[4 P_{3,3}-2 P_{3,4}-2 P_{4,3}\right],
$$

which contradicts Lemma 4.2.

Case 2. Suppose that $c_{4}=0$. Then, by the relations in Section 1 , we get

$$
0=D=\left[2\left(P_{1,1}+P_{1,2}+P_{1,3}+P_{2,3}\right)-8 P_{3,3}\right]=\left[2 P_{2,3}-2 P_{1,4}\right],
$$

which implies the existence of a morphism $\mathcal{C} \rightarrow \mathbb{P}^{1}$ of degree 2 , contrary to the fact that $\mathcal{C}$ is not hyperelliptic.

Case 3. Suppose that $c_{2}=0$. Then, by the relations in Section 1, we get

$$
0=D=\left[2\left(P_{1,1}+P_{1,3}+P_{2,1}+P_{2,3}\right)-8 P_{3,3}\right]=\left[2 P_{1,1}+2 P_{2,1}-2 P_{3,3}-2 P_{3,4}\right] .
$$

Let $f$ be a rational function on $\mathcal{C}$ such that $\operatorname{div}(\mathrm{f})=2 \mathrm{P}_{1,1}+2 \mathrm{P}_{2,1}-2 \mathrm{P}_{3,3}-2 \mathrm{P}_{3,4}$. If $E=2 P_{3,3}+2 P_{3,4}$ and $K$ is a canonical divisor on $\mathcal{C}$, note that $E$ is not linearly equivalent to $K$ and $\operatorname{deg}(K-E)=0$. Therefore, the vector space $L(K-E)$ is zero-dimensional. Hence, by the Riemann-Roch theorem, the vector space $L(E)$ is two-dimensional. If $\infty_{j}, 1 \leq j \leq 4$, are the four points of intersection of $\mathcal{C}$ with the line $Z=0$, then it is straightforward to show that

$$
\begin{gathered}
\operatorname{div}\left(\mathrm{x}^{2}+\mathrm{y}^{2}+2\right)=2 \mathrm{P}_{3,3}+2 \mathrm{P}_{3,4}+2 \mathrm{P}_{4,3}+2 \mathrm{P}_{4,4}-\sum_{\mathrm{j}=1}^{4} 2 \infty_{\mathrm{j}}, \\
\operatorname{div}(\mathrm{y}+\mathrm{i})=\mathrm{P}_{1,4}+\mathrm{P}_{2,4}+\mathrm{P}_{4,3}+\mathrm{P}_{4,4}-\sum_{\mathrm{j}=1}^{4} \infty_{\mathrm{j}} .
\end{gathered}
$$

It follows that $L(E)$ is generated by the rational functions 1 and $(y+i)^{2} /\left(x^{2}+y^{2}+2\right)$. In particular, $f$ can be taken to be of the form

$$
f=\frac{a\left(x^{2}+y^{2}+2\right)+(y+i)^{2}}{x^{2}+y^{2}+2}
$$

for some constant $a \in \mathbb{C}$. Since $P_{1,1}$ is a zero of $f$, we must have $a=-i$. However, for this choice of $a$, the point $(i \sqrt{5},-1,1)$ is also a zero of $f$, a contradiction.

\section{ACKNOWLEDGMENTS}

The first author would like to thank her advisor, Marc Hindry, for all his precious suggestions. Both authors would like to thank David Rohrlich for making this collaboration possible. 


\section{REFERENCES}

[1] Martine Girard, Groupe des points de Weierstrass sur une famille de quartiques lisses, Preprint, December 1999.

[2] Matthew J. Klassen and Edward F. Schaefer, Arithmetic and geometry of the curve $y^{3}+1=x^{4}$, Acta Arith. 74 (1996), no. 3, 241-257. MR 96k:11081

[3] Leopoldo Kulesz, Courbes elliptiques de rang $\geq 5 \operatorname{sur} \mathbb{Q}(t)$ avec un groupe de torsion isomorphe $\grave{a} \mathbb{Z} / 2 \mathbb{Z} \times \mathbb{Z} / 2 \mathbb{Z}$, C. R. Acad. Sci. Paris Sér. I Math. 329 (1999), no. 6, 503-506. MR 2000f:11068

[4] Akikazu Kuribayashi and Kaname Komiya, On Weierstrass points and automorphisms of curves of genus three, Algebraic geometry (Proc. Summer Meeting, Univ. Copenhagen, Copenhagen, 1978), Springer, Berlin, 1979, pp. 253-299. MR 81c:14016

[5] David Mumford, Tata lectures on theta. II, Birkhäuser Boston Inc., Boston, MA, 1984, Jacobian theta functions and differential equations. MR 86b:14017

[6] Despina T. Prapavessi, On the Jacobian of the Klein curve, Proc. Amer. Math. Soc. 122 (1994), no. 4, 971-978. MR 95b:14023

[7] David E. Rohrlich, Points at infinity on the Fermat curves, Invent. Math. 39 (1977), no. 2, 95-127. MR 56:367

Théorie des Nombres, Institut de Mathématiques de Jussieu, 175, Rue du Chevaleret, 75013 PARIS, France

E-mail address: girard@math.jussieu.fr

Department of Mathematics, The University of Arizona, P.O. Box 210089, 617 N. Santa Rita, Tucson, Arizona 85721-0089

E-mail address: tzermias@math.arizona.edu

Current address: Department of Mathematics, University of Tennessee, Knoxville, Tennessee 37996-1300

E-mail address: tzermias@math.utk.edu 